\title{
Elevated Circulating Acylated and Total Ghrelin Concentrations Along With Reduced Appetite Scores in Infants With Failure to Thrive
}

\author{
GLORIA SHAFFER TANNENBAUM, MARIA RAMSAY, CHANTAL MARTEL, MARWAN SAMIA, \\ CATHERINE ZYGMUNTOWICZ, MAFALDA PORPORINO, AND SHUVO GHOSH
}

\begin{abstract}
Department of Pediatrics [G.S.T., M.R., S.G.], Department of Neurology and Neurosurgery [G.S.T.], Neuropeptide Physiology Laboratory [G.S.T., M.S.], Department of Psychology [M.R., C.M., C.Z., M.P.], McGill University and the Montreal Children's Hospital Research Institute, Montreal, Québec H3H 1P3, Canada
\end{abstract}

\begin{abstract}
Failure to thrive (FTT) is a term used to describe inadequate growth in infants. The immediate cause is undernutrition. Ghrelin is a potent orexigenic hormone that induces a positive energy balance and enhances appetite. There is no information regarding the possible role of ghrelin in infants with FTT. The aim of this study was 2-fold: 1) to examine circulating ghrelin levels in FTT infants, compared with those of normally growing infants; and 2) to evaluate appetitive behaviors in the two groups. Plasma acylated and total ghrelin concentrations were measured in nine FTT and five normally growing infants (age range, 9-18 mo). Appetite was assessed using three novel appetite measures. Both acylated and total ghrelin levels were significantly elevated in FTT infants compared with controls ( $p=0.03$ or less). Infants with FTT scored significantly lower than control infants on all appetite measures ( $p=0.002$ or less). Ghrelin levels were inversely related to appetite, weight velocity, weight/ length $z$-scores, and weight $z$-score. These findings provide the first evidence that infants with FTT have higher circulating ghrelin concentrations but paradoxically lower appetite scores. Increased ghrelin secretion may reflect an adaptive mechanism attempting to increase appetite and preserve energy balance in response to poor nutritional state. (Pediatr Res 65: 569-573, 2009)
\end{abstract}

$F^{a}$ ailure to thrive (FTT) in infancy occurs when an infant's weight gain deviates at least two major percentiles downward on a standardized growth chart, crossing below the 5th percentile (1). The immediate cause of this pediatric condition is undernutrition, which may be secondary to medical causes (2) or to the infant's inability or unwillingness to ingest an adequate amount of nutrients (3). Undernutrition need not be severe or prolonged to produce behavioral changes in young children (4) and, when prolonged, it may influence brain development and produce cognitive, motor, and neurologic deficits during the critical period of rapid brain growth (5). In the absence of medical disorders to explain undernutrition in infants with FTT, parenting behaviors have been associated with the infant's poor growth (see 6 for review). However, the

Received May 9, 2008; accepted November 10, 2008

Correspondence: Gloria S. Tannenbaum, Ph.D., McGill University-Montreal Children's Hospital Research Institute, 2300 Tupper Street, Montreal, Québec H3H 1P3, Canada; e-mail: gloria.tannenbaum@mcgill.ca

Supported by the Canadian Institutes of Health Research [grant MOP 64328 (G.S.T.)]. G.S.T. holds a Career Investigator Award from the Fonds de la recherche en santé du Québec.

This article contains supplementary material available online at www.pedresearch.org. possibility that FTT may involve an underlying appetite problem has been given little attention until recently. Wright and Birks (7) reported that children with FTT were described by their parents as being uninterested in food and as poor or variable eaters. Clinical signs of low appetite have also been previously reported by us (3); however, systematic assessment of appetitive behaviors and their possible underlying hormonal correlates have not been documented in infants with FTT.

Ghrelin is a potent orexigenic hormone and growth hormone $(\mathrm{GH})$ secretagogue that is predominantly produced in the stomach but is also present in the hypothalamic arcuate nucleus $(8,9)$. It is a novel 28 -amino acid peptide that circulates in two major forms: $n$-octanoylated ghrelin, in which a hydroxyl group at its serine 3 residue is acylated by $n$-octanoic acid, and nonacylated (des-acyl) ghrelin; acylation seems to be essential for ghrelin's biologic activities (10). Ghrelin strongly stimulates $\mathrm{GH}$ release in both animals $(8,11)$ and humans (12) and induces a positive energy balance leading to body weight gain (13) independent of its GH-releasing activity. In humans, exogenous ghrelin enhances appetite and increases subjective hunger/food intake in healthy individuals (14). A preprandial rise of human plasma ghrelin suggests a possible role of ghrelin as a hunger signal triggering meal initiation (15), and studies in patients with different eating disorders show that circulating ghrelin levels are perturbed in anorexia nervosa (16), bulimia (17) and obesity (18). An inverse relationship between ghrelin concentration and weight gain has also been documented in healthy term infants (19).

Although evidence supports the notion that ghrelin may serve as an important modulator of appetite in animals and human adults there is no report, to our knowledge, regarding the possible role of ghrelin in infants with FTT. Circulating ghrelin levels and their relationship with appetite have yet to be documented in FTT infants. Although visual analogue scales and questionnaires have been used to measure appetite in adults (14) and adolescents (20), to date there is no reported measure of appetitive behaviors in preverbal infants. There-

Abbreviations: ACS, Appetite Composite Score, FTT, Failure to thrive, MCH-FS, Montreal Children's Hospital Feeding Scale 
fore, the aims of this study were 1) to investigate circulating ghrelin levels, both the acylated and total forms, in infants with FTT compared with those of normally growing infants and 2) to assess appetitive behaviors in the two groups using a newly developed appetite measure. The relationships between appetite levels, ghrelin concentrations, anthropometric and hormonal growth-promoting parameters (GH and IGF-1) were also examined.

\section{METHODS}

Subjects. The study included nine infants with FTT (all crossed at least two major percentiles on the standardized CDC/NCHS 2000 growth chart over at least three consecutive months and at the time of assessment were under the 5th percentile) and five comparison infants with normal growth (all growing steadily with weight above the 50th percentile on the growth chart during a 3-mo period). Infants with FTT and two comparison infants were recruited from the Failure to Thrive and Feeding Disorders (FTT/FD) Clinic of the Montreal Children's Hospital. Subjects with FTT were referred by their pediatricians for poor weight gain and suspected inadequate food intake; the infants with normal growth were referred for minor sensitivities to highertextured foods. None of the nine FTT infants enrolled in the study had a medical condition to explain their poor growth. The two comparison infants with normal growth were also without medical condition; their minor sensitivities to food textures resulted from inexperience with higher-textured foods. Three other comparison infants with normal growth were recruited from the Endocrinology Clinic of the same hospital. These infants had a history of congenital hypothyroidism and were treated with L-thyroxine; all three were euthyroid for at least 8 mo before study. Several reports have shown that once hypothyroidism is treated, circulating ghrelin levels do not differ from those of healthy controls (21). All infants had regular weight monitoring by their pediatricians and there was no significant difference in weight $z$-scores between control infants recruited from the two different clinics. At the time of assessment, infants with FTT were between 10 and 15 mo of age and normally growing infants were between 9 and 18 mo. The Institutional Review Board of the Montreal Children's Hospital approved the study protocol and written informed consent was obtained from all parents.

Procedure. All families underwent a semistructured clinical interview where anthropometric measures and a detailed developmental and feeding history were obtained. Before the interview, parents completed a feeding scale [The Montreal Children's Hospital Feeding Scale (MCH-FS), see later] and a general background information questionnaire. Parents and their infants were observed during a meal in the clinic. All medical charts were reviewed by the clinic pediatrician. Participants recruited through the FTT/FD Clinic returned within 1 wk after the initial assessment to have blood samples collected. For subjects recruited through the Endocrinology Clinic, blood was obtained as part of the routine blood work that included a complete blood count and an electrolyte panel. Parents were asked to refrain from feeding their child at least $3 \mathrm{~h}$ before the blood collection. As six of the 14 infants were still breast-fed (or breast and bottle-fed) in addition to solid food, caloric intake could not be calculated. The technician collecting the blood samples and measuring ghrelin levels was unaware of the grouping of the infants.

Appetite measures. Three sources were used for assessing appetitive behaviors in infants:

The Montreal Children's Hospital Feeding Scale. This is a 14-item parent-report instrument where each appetite-related item is rated on a Likert scale from 1 to 7 (see Supplemental Questionnaire). Three items in this scale reflect infants' appetite: item no. 3 (how much appetite does your child have?), no. 4 (when does your child start refusing to eat during mealtimes?), and no. 9 (do you have to follow your child around or use distractions so that your child will eat?). The mean score of the three items for each subject ranged from 1 to 7 . The MCH-FS has been validated on a general pediatric population in the community $(n=200)$ and a referred clinic population $(n=$ $180)$. The results indicated excellent discriminate validity $(p<0.001)$ and test-retest reliability for the individual items $(r=0.76-0.97)$.

Appetite History Scale. The Appetite History Scale covers the period from birth to $2 \mathrm{y}$ and includes four appetitive behaviors: signaling hunger, consuming age-appropriate portions, concentrating during feeds, feeding in less than $30 \mathrm{~min}$. These items were rated by parents as mostly absent (score 0 ) or mostly present (score 1), and weighted to give a total score from 0 to 7 . The infant's recent appetitive behaviors (during 1-6 mo before assessment) were based on parental responses to questions by the interviewer. Validity of this scale was established in the clinic on 10 children growing normally and 22 children with FTT; the mean \pm SD score was $6.30 \pm 1.35$ for the normally growing infants and $1.05 \pm 1.70$ for infants with FTT, at $p=0.0001$ level.

Observed Feeding Behaviors Scale. The observed feeding behaviors in the clinic at the beginning $(5-10 \mathrm{~min})$ of a meal with preferred food after at least $3 \mathrm{~h}$ of food deprivation were scored as either 1 (problematic feeder), 2 (passive feeder), or 3 (active feeder). Observer interrater reliability of $r=1.0$ has been established on 20 subjects in the clinic.

An overall Appetite Composite Score (ACS) was derived for each subject by summing their scores obtained on the above three measures. The ACS ranged from 2 to 17 .

Plasma collection. All blood collections took place between 0900 and $1200 \mathrm{~h}$; the times of blood collection were similar in FTT and comparison infants. Approximately $3 \mathrm{~mL}$ of blood were obtained from each patient through venipuncture and collected in Vacutainer tubes (Becton Dickinson) containing potassium EDTA. The blood samples were immediately stored on ice until they were centrifuged at $4^{\circ} \mathrm{C}$ for $20 \mathrm{~min}$ at $1200 \times \mathrm{g}$. Plasma was separated and divided into two aliquots: the aliquot for subsequent ghrelin measurement was treated with $1 \mathrm{~N} \mathrm{HCl}(50 \mu \mathrm{L} / \mathrm{mL}$ of plasma) and $10 \mathrm{mg} / \mathrm{mL}$ phenylmethylsulfonyl fluoride (PMSF) dissolved in isopropanol $(10 \mu \mathrm{L} / \mathrm{mL}$ of plasma) to preserve the octanoyl moiety on ghrelin, as recommended by the manufacturer of the ghrelin assay kits (Linco Research, St. Louis, MI). The acidified plasma was stored at $-80^{\circ} \mathrm{C}$ for subsequent assay of total and acylated ghrelin. The other aliquot was stored at $-80^{\circ} \mathrm{C}$ for subsequent assay of GH and IGF-1.

Assays. Plasma ghrelin concentrations were measured in duplicate using two commercially available double antibody RIAs (Linco Research, St. Louis, MI; catalogue numbers: GHRA-88HK and GHRT-89HK) that distinguish the intact octanoylated (active) from nonoctanoylated ghrelin. The antiserum used in the active RIA specifically recognizes acylated ghrelin (ghrelin 1-10), but does not recognize des-acyl ghrelin or the ghrelin 14-28 fragment, whereas the antiserum used in the second assay equally recognizes acylated and des-acyl ghrelin (i.e. total). Neither antiserum exhibits significant crossreactivity with other peptides, including leptin, insulin, glucagon, and motilinrelated peptide. The minimum detectable levels of acylated and total ghrelin were $10 \mathrm{pg} / \mathrm{mL}$ and $100 \mathrm{pg} / \mathrm{mL}$, respectively. In both assays, the intra and interassay variations were less than $10 \%$ and $17 \%$, respectively. To minimize variability, all samples for acylated and total ghrelin were measured in single assays, respectively.

Plasma GH and IGF-1 concentrations were measured in duplicate by double antibody RIA (22) using materials supplied by the NIDDK Hormone Distribution Program (Bethesda, MD). The averaged plasma GH values are reported in terms of the human GH reference preparation (hGH-RP-1). The standard curve was linear between 1.0 and $100 \mathrm{ng} / \mathrm{mL}$. The intra and interassay coefficients of variation were $5.1 \%$ and $8.9 \%$, respectively. The averaged plasma IGF-1 values are reported in terms of the human IGF-1 reference preparation (Lot \# 01). The standard curve was linear between 0.2 and $25 \mathrm{ng} / \mathrm{mL}$. The intra and interassay coefficients of variation were $12.8 \%$ and $17.9 \%$, respectively. All samples for GH and IGF-1 were measured in single assays, respectively.

Data analyses. Ponderal Index for birth weight was calculated for each subject using the following formula: weight $(\mathrm{g}) /$ length $\left(\mathrm{cm}^{2}\right) \times 100$. Weight, length, and weight/length $z$-scores were calculated using the nutrition program of EpiInfo version 3.3.2. For each subject, multiple body weight measures were obtained from the health book records kept by their pediatrician. Starting at 6 mo of age and until the time of assessment, these measures were regressed against age using a logarithmic model to account for the nonlinear growth in that age range. The weight velocity reported represents the coefficient of the age variable in the regression equations generated. All logarithmic regressions were done using MS-Excel. Lillifore's test was used to verify the normality of all continuous variables. Statistical comparisons between the groups were performed using $t$ tests for normally distributed variables and Mann-Whitney $U$-tests for nonnormally distributed variables. The Pearson product-moment correlation coefficient was used to evaluate the degree of relation among the various continuous variables. Results are expressed as the mean \pm SE; $p<0.05$ was considered significant. SYSTAT 9 and SigmaPlot 8 were used for statistical analyses and data presentation, respectively. The observed power of the various statistical tests was calculated using SigmaStat 3.5 and ranged between 0.73 and 1.00 .

\section{RESULTS}

Subjects. The characteristics of the participants are described in Table 1. There were no significant differences between infants with FTT and controls in gestational age, birth weight, Ponderal Index at birth, or in their age at the time of 
Table 1. Characteristics of the FTT infants compared with controls

\begin{tabular}{lrcc}
\hline & FTT $(n=9)$ & Control $(n=5)$ & $p$ \\
\hline Sex (M/F) & $4 / 5$ & $3 / 2$ & \\
Gestational age (wk) & $38.44 \pm 0.75$ & $39.60 \pm 0.75$ & 0.3367 \\
Birth weight (kg) & $2.92 \pm 0.17$ & $3.29 \pm 0.33$ & 0.2768 \\
Ponderal index at birth & $2.48 \pm 0.16$ & $2.58 \pm 0.11$ & 0.6840 \\
Weight (z-score) at 6 mo & $-0.91 \pm 0.25$ & $0.18 \pm 0.51$ & 0.0510 \\
Assessment time: age (mo) & $12.85 \pm 0.63$ & $12.72 \pm 1.51$ & 0.9263 \\
Weight (z-score) & $-2.10 \pm 0.20$ & $0.60 \pm 0.26$ & 0.0001 \\
Length (z-score) & $-1.10 \pm 0.18$ & $0.15 \pm 0.26$ & 0.0018 \\
Weight/Length (z-score) & $-1.73 \pm 0.20$ & $0.71 \pm 0.18$ & 0.0027 \\
Weight velocity (kg/ln mo) & $1.49 \pm 0.07$ & $3.85 \pm 0.43$ & 0.0001 \\
\hline
\end{tabular}

Data represent the mean $\pm \mathrm{SE}$.

the study. However, by 6 mo of age, infants in the FTT group already had lower weight $z$-scores than their comparison infants and, at the time of assessment, weight, height, and weight/height $z$-scores of the FTT group were severely decreased compared with the normally growing control group (Table 1). Infants in the FTT group gained weight at a 2- to 3 -fold slower rate than controls in the period ranging from 6 mo of age until assessment date (mean weight velocity: $1.49 \pm 0.07$ vs. $3.85 \pm 0.43 \mathrm{~kg} / \mathrm{ln} \mathrm{mo} ; p<0.0001$ ). All infants in this study had age-appropriate developmental skills and no psychosocial issues were detected during the initial assessment. For all infants with FTT, the standard nutritional panel and complete blood count were within normal limits, and thus no further medical investigations were deemed necessary.

Appetite scores. Infants with FTT scored significantly lower (2- to 15-fold) than normally growing control infants on all three measures used to assess appetite (Fig. 1). The mean ACS of the FTT group at time of assessment was 3- to 4-fold lower compared with that observed in control infants $(p<0.002)$. Of interest, the mean score on the Appetite History Scale from birth to 6 mo for infants with FTT was already significantly lower than that for their comparison group (1.67 vs. 7.00; $p<$ 0.0001).

Acylated and total plasma ghrelin concentrations. Figure 2 illustrates the mean plasma total and acylated ghrelin concen-

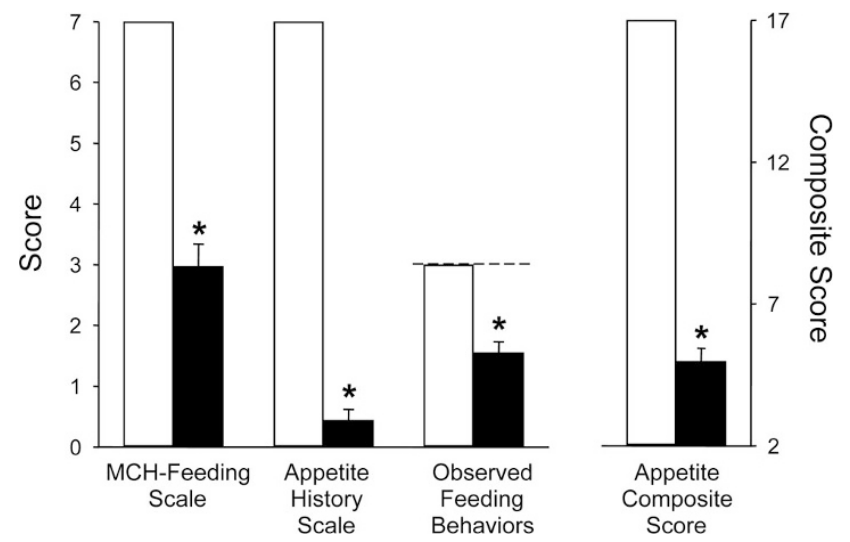

Figure 1. Appetite scores on three different measures, and composite score in nine infants with FTT (ם) compared with five normally growing control infants $(\square)$. Each bar represents the mean \pm SE. Dashed line indicates upper limit of Observed Feeding Behaviors Scale. ${ }^{*} p=0.002$ or less $v s$. control infants.

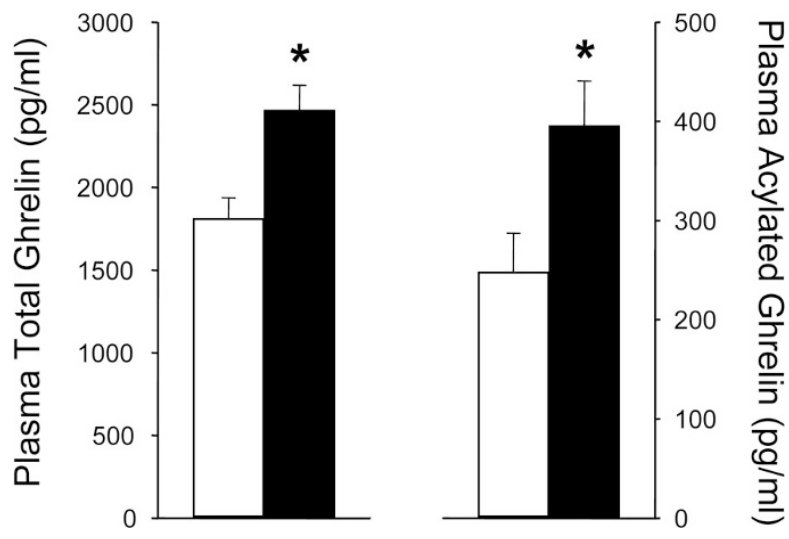

Figure 2. Total and acylated plasma ghrelin levels in nine FTT infants ( $\square$ compared with five normally growing controls $(\square)$. Both total and acylated ghrelin were significantly augmented in infants with FTT compared with control infants. Each bar represents the mean $\pm \mathrm{SE} * p=0.03$ or less $v s$. controls.

trations in infants with FTT compared with controls. Both acylated and total ghrelin levels were significantly elevated in FTT subjects compared with those observed in normally growing control infants (acylated: $396 \pm 45$ vs. $248 \pm 39$ $\mathrm{pg} / \mathrm{mL} ; p=0.012 ;$ total: $2469 \pm 149 v s .1812 \pm 125 \mathrm{pg} / \mathrm{mL}$; $p=0.038)$. Acylated ghrelin concentrations comprised approximately $13-17 \%$ of total circulating ghrelin in the two groups. The ratio of acylated to total ghrelin was somewhat higher in FTT infants compared with controls $(17.5 \pm 1.4 \%$ vs. $13.4 \pm 1.3 \%$ ), but this difference did not reach significance.

Plasma GH and IGF-1 levels. Mean plasma GH levels in infants with FTT $(5.5 \pm 1.3 \mathrm{ng} / \mathrm{mL})$ were not significantly different $(p=0.99)$ from those observed in control subjects $(5.4 \pm 2.6 \mathrm{ng} / \mathrm{mL})$. Although mean plasma IGF-1 levels were lower in FTT infants than in comparison infants, no significant difference was identified $(33.0 \pm 10.5 v s .47 .1 \pm 21.3 \mathrm{ng} / \mathrm{mL}$; $p=0.53)$.

Relationships between ghrelin, appetite, anthropometric, and hormonal measures. Table 2 summarizes the associations between hormones, appetite, and anthropometric parameters. The two forms of ghrelin were strongly positively associated $(p<0.0001)$. Total ghrelin levels were inversely related to both ACS and weight/length $z$-score, as well as weight velocity and weight $z$-score. Acylated ghrelin levels were negatively correlated with both appetite measures and weight velocity. No significant correlations were noted between either total or acylated ghrelin values and age, gender or feeding mode, as well as GH or IGF-1 levels. A strong positive correlation was observed between GH and IGF-1 ( $r=0.74$, $p<0.006$ ), whereas no significant associations were found between GH and IGF-1 and all other parameters. A strong positive relationship was noted among the three appetite measures $(r=0.72-0.94, p<0.003$ or less). Weight velocity was positively related to both ACS $(r=0.89, p<$ $0.0001)$ and weight/length $z$-scores $(r=0.85, p<0.0001)$.

\section{DISCUSSION}

This study is the first to demonstrate that infants with FTT have significantly higher circulating levels of both acylated 
Table 2. Relationships between circulating levels of ghrelin, appetite, anthropometric, and hormonal measures in 14 FTT and control infants

\begin{tabular}{|c|c|c|c|c|c|c|}
\hline & \multicolumn{2}{|c|}{ Total ghrelin } & \multicolumn{2}{|c|}{ Acylated ghrelin } & \multicolumn{2}{|c|}{$\begin{array}{l}\text { Appetite composite } \\
\text { score }\end{array}$} \\
\hline & $r$ & $p$ & $r$ & $p$ & $r$ & $p$ \\
\hline Age & -0.21 & 0.475 & -0.33 & 0.367 & -0.10 & 0.738 \\
\hline Gender & -0.24 & 0.418 & 0.44 & 0.209 & -0.17 & 0.559 \\
\hline Feeding mode & 0.25 & 0.395 & -0.30 & 0.412 & 0.19 & 0.523 \\
\hline Appetite history scale & -0.65 & 0.011 & -0.63 & 0.049 & 0.99 & 0.000 \\
\hline MCH-feeding scale & -0.71 & 0.004 & -0.55 & 0.099 & 0.96 & 0.000 \\
\hline Observed feeding behaviors & -0.42 & 0.133 & -0.67 & 0.034 & 0.86 & 0.000 \\
\hline Appetite composite score & -0.67 & 0.009 & -0.63 & 0.052 & & \\
\hline Weight velocity (preassessment time) & -0.64 & 0.014 & -0.70 & 0.024 & 0.89 & 0.000 \\
\hline Weight $z$-score & -0.55 & 0.044 & -0.40 & 0.253 & 0.92 & 0.000 \\
\hline Weight/length $z$-score & -0.66 & 0.009 & -0.50 & 0.147 & 0.93 & 0.000 \\
\hline Total ghrelin & & & 0.95 & 0.000 & -0.67 & 0.009 \\
\hline $\mathrm{GH}$ & 0.22 & 0.444 & -0.32 & 0.368 & -0.02 & 0.958 \\
\hline IGF-1 & -0.42 & 0.176 & -0.22 & 0.544 & 0.24 & 0.454 \\
\hline
\end{tabular}

and total ghrelin than normally growing control infants. Furthermore, the results reported here provide the first evidence that both forms of ghrelin are inversely correlated with appetite in this pediatric age group (under $2 \mathrm{y}$ of age).

The finding of elevated ghrelin concentrations in infants with FTT provides additional support for the concept that circulating ghrelin levels may be a reliable marker of nutritional status (23). In our study, the nutritional states of infants in both groups were similar at birth, as indicated by their Ponderal Index; however, infants in the FTT group progressively failed to gain weight age-appropriately, particularly from 6 mo of age, resulting in their malnutritioned state at the time of assessment, as evidenced by their markedly lower anthropometric scores when compared with the group displaying normal growth. Ghrelin concentrations were found to be inversely correlated with several growth-related anthropometric measures, including weight velocity and weight/length $z$-scores. These findings are consistent with previous studies showing elevated umbilical cord and blood ghrelin concentrations in neonates who were small for their gestational age and thus were in a state of undernutrition compared with neonates who were appropriate for gestational age or large for gestational age $(24,25)$. They are also in accord with observations in adolescents and young adults exhibiting anorexia nervosa reflecting a state of negative energy balance $(16,20)$.

We originally hypothesized that ghrelin levels may be altered in children with FTT and a history of poor appetite, thereby pointing to a possible role for ghrelin in the etiology of this disorder. Our finding, however, of elevated (rather than reduced) plasma levels of both acylated and total ghrelin makes it unlikely that alterations in ghrelin secretion are directly responsible for the pathogenesis of FTT. Although ghrelin does not seem to play a causative role in FTT, increased bioactive ghrelin secretion in FTT infants may reflect an adaptive mechanism signaling the hypothalamus to increase appetite and preserve energy balance in response to their poor nutritional state. These patients with poor appetite, however, appear to be insensitive to the orexigenic actions of ghrelin. Desensitization of the hypothalamic ghrelin receptor, GHS-R, implicated in the control of food intake could explain in part the paradoxical response of increased ghrelin and decreased feeding leading to a malnutritioned state in FTT. Indeed, prolonged exposure to hyperghrelinemia in patients with anorexia nervosa has been reported to result in a blunted response to the actions of exogenous ghrelin (26). Moreover, both ghrelin receptor mRNA levels (27) and ghrelin gene expression and peptide content (9) in hypothalamus were shown to be markedly altered in response to perturbed nutritional states such as fasting. Further study will be required to address the issue of ghrelin receptor(s) sensitivity mechanisms in the hypothalamus as a possible mechanism underlying the poor appetite in FTT.

This study is the first to report a systematic evaluation of appetite in nonverbal infants under $2 \mathrm{y}$ of age using maternal report of appetitive behaviors combined with observed feeding behaviors during a mealtime. As there are no known objective assessment tools available for those who cannot communicate their hunger verbally, the present ACS is a unique and, to the best of our knowledge, the only known attempt to identify appetite levels in infants. Although an important limitation of this study is the small number of infants investigated, the ability to detect significant differences despite the small sample size further supports the magnitude of the findings. The difficulty in studying appetitive behaviors in this young age group should also be recognized. Although we used parental report of infant appetitive behaviors, the inclusion of two forms of parental report as well as observation of feeding behaviors by a clinician likely mitigated possible recall bias, particularly because the three forms of appetite assessment correlated very highly.

Our data showed an inverse relationship between appetite and circulating ghrelin concentrations. At the time of assessment, appetite scores were markedly lower (2- to 15-fold) in the FTT group compared with their normally growing controls on all three measures, suggesting that low appetite is one of the characteristics of the FTT group. Of interest, although the levels of appetite in FTT infants were consistently lower than in the comparison group since birth, as reported by parents in the Appetite History Scale, their weights at birth were similar but by 6 mo were somewhat lower than in control infants and 
significantly lower at the time of assessment. These results suggest that poor appetite likely predates the malnutritioned state in infants with FTT. Although Wren et al. (6) have shown that ghrelin infusion stimulated appetite and increased food intake in normal human subjects, it is unlikely, based on the results of the present study, that increasing an already high plasma ghrelin level by giving ghrelin exogenously will have orexigenic effects in infants with FTT. Indeed, ghrelin infusions in patients with anorexia nervosa did not affect appetite (28).

Plasma GH and IGF-1 levels in infants with FTT were not significantly different from normally growing infants. In this regard, it should be noted that a single blood sample may not accurately reflect GH status; however, frequent blood sampling could not be performed because of sampling volume constraints in this pediatric age group. Although IGF-1 levels were lower in the FTT group compared with controls, consistent with IGF-1 being diminished in malnourished states (22), this difference did not attain significance. This finding is similar to that previously reported in young children with nonorganic FTT (29). We also did not observe any significant correlations between plasma acylated or total ghrelin and $\mathrm{GH}$ or IGF-1 levels. This lack of relationship between ghrelin and GH/IGF-1 is in agreement with several other studies $(30,31)$ suggesting that ghrelin's primary role may be in regulating energy homeostasis with only an ancillary role as a $\mathrm{GH}$ secretagogue.

In summary, our results demonstrate that both acylated and total plasma ghrelin concentrations are significantly elevated in infants with FTT and low appetite, compared with infants displaying normal growth. They further show that circulating ghrelin levels are inversely correlated with appetite and growth-related anthropometric measures but are not associated with either GH or IGF-1 levels. Measured appetitive behaviors discriminate between infants with FTT and those growing normally as early as 3-6 mo before their diagnosis of FTT, suggesting that appetitive behaviors contribute to inadequate intake and poor growth in this population. Increased ghrelin secretion in FTT infants may reflect an adaptive mechanism by their body attempting to increase appetite and preserve positive energy balance in response to poor nutritional status; such hyperghrelinemia could lead to desensitization of the hypothalamic GHS-R receptor(s) implicated in the control of food intake. An additional study with a larger number of infants will be needed to confirm these findings.

Acknowledgments. We thank the infants and their parents for participating in this study. We thank Dr. Celia Rodd for assistance in recruiting control children, the NIDDK and Dr. A. F. Parlow for the provision of GH and IGF-1 RIA materials, and Zehra J. Khoja for preparation of the manuscript.

\section{REFERENCES}

1. Casey PH 1996 Failure to thrive. In: Levine MD, Carey WB, Crocker AC (eds) Developmental Behavioral Pediatrics. Saunders Co., Toronto, pp 397-405

2. Bithoney WG, Dubowitz H, Egan H 1992 Failure to thrive/growth deficiency. Pediatr Rev 13:453-460

3. Ramsay M, Gisel EG, Boutry M 1993 Non-organic failure to thrive: growth failure secondary to feeding-skills disorder. Dev Med Child Neurol 35:285-297
4. Lozoff B 1989 Nutrition and behavior. Am Psychol 44:231-236

5. Corbett SS, Drewett RF 2004 To what extent is failure to thrive in infancy associated with poorer cognitive development? A review and meta-analysis. J Child Psychol Psychiatry 45:641-654

6. Boddy JM, Skuse DH 1994 The process of parenting in failure to thrive. J Child Psychol Psychiatry 35:401-424

7. Wright C, Birks E 2000 Risk factors for failure to thrive: a population-based survey Child Care Health Dev 26:5-16

8. Kojima M, Hosoda H, Date Y, Nakazato M, Matsuo H, Kangawa K 1999 Ghrelin is a growth-hormone-releasing acylated peptide from stomach. Nature 402:656-660

9. Sato T, Fukue Y, Teranishi H, Yoshida Y, Kojima M 2005 Molecular forms of hypothalamic ghrelin and its regulation by fasting and 2-deoxy-D-glucose administration. Endocrinology 146:2510-2516

10. Hosoda H, Kojima M, Matsuo H, Kangawa K 2000 Ghrelin and des-acyl ghrelin: two major forms of rat ghrelin peptide in gastrointestinal tissue. Biochem Biophys Res Commun 279:909-913

11. Tannenbaum GS, Epelbaum J, Bowers CY 2003 Interrelationship between the novel peptide ghrelin and somatostatin/growth hormone-releasing hormone in regulation of pulsatile growth hormone secretion. Endocrinology 144:967-974

12. Takaya K, Ariyasu H, Kanamoto N, Iwakura H, Yoshimoto A, Harada M, Mori K, Komatsu Y, Usui T, Shimatsu A, Ogawa Y, Hosoda K, Akamizu T, Kojima M, Kangawa K, Nakao K 2000 Ghrelin strongly stimulates growth hormone (GH) release in humans. J Clin Endocrinol Metab 85:4908-4911

13. Tschöp M, Smiley DL, Heiman ML 2000 Ghrelin induces adiposity in rodents. Nature 407:908-913

14. Wren AM, Seal LJ, Cohen MA, Brynes AE, Frost GS, Murphy KG, Dhillo WS, Ghatei MA, Bloom SR 2001 Ghrelin enhances appetite and increases food intake in humans. J Clin Endocrinol Metab 86:5992-5995

15. Cummings DE, Purnell JQ, Frayo RS, Schmidova K, Wisse BE, Weigle DS 2001 A preprandial rise in plasma ghrelin levels suggests a role in meal initiation in humans. Diabetes 50:1714-1719

16. Otto B, Cuntz U, Fruehauf E, Wawarta R, Folwaczny C, Riepl RL, Heiman ML, Lehnert P, Fichter M, Tschop M 2001 Weight gain decreases elevated plasma ghrelin concentrations of patients with anorexia nervosa. Eur J Endocrinol 145:669673

17. Tanaka M, Naruo T, Muranaga T, Yasuhara D, Shiiya T, Nakazato M, Matsukura S, Nozoe S 2002 Increased fasting plasma ghrelin levels in patients with bulimia nervosa. Eur J Endocrinol 146:R1-R3

18. Tschöp M, Weyer C, Tataranni PA, Devanarayan V, Ravussin E, Heiman ML 2001 Circulating ghrelin levels are decreased in human obesity. Diabetes 50:707-709

19. Savino F, Liguori SA, Fissore MF, Oggero R, Silvestro L, Miniero R 2005 Serum ghrelin concentration and weight gain in healthy term infants in the first year of life. J Pediatr Gastroenterol Nutr 41:653-659

20. Stock S, Leichner P, Wong AC, Ghatei MA, Kieffer TJ, Bloom SR, Chanoine J-P 2005 Ghrelin, peptide YY, glucose-dependent insulinotropic polypeptide, and hunger responses to a mixed meal in anorexic, obese, and control female adolescents. J Clin Endocrinol Metab 90:2161-2168

21. Gjedde S, Vestergaard ET, Gormsen LC, Riis AL, Rungby J, Moller N, Weeke J, Jorgensen JO 2008 Serum ghrelin levels are increased in hypothyroid patients and become normalized by L-thyroxine treatment. J Clin Endocrinol Metab 93:22772280

22. Harel Z, Tannenbaum GS 1995 Long-term alterations in growth hormone and insulin secretion after temporary dietary protein restriction in early life in the rat. Pediatr Res 38:747-753

23. Saper CB, Chou TC, Elmquist JK 2002 The need to feed: homeostatic and hedonic control of eating. Neuron 36:199-211

24. Farquhar J, Heiman M, Wong AC, Wach R, Chessex P, Chanoine JP 2003 Elevated umbilical cord ghrelin concentrations in small for gestational age neonates. J Clin Endocrinol Metab 88:4324-4327

25. Kitamura S, Yokota I, Hosoda H, Kotani Y, Matsuda J, Naito E, Ito M, Kangawa K, Kuroda Y 2003 Ghrelin concentration in cord and neonatal blood: relation to feta growth and energy balance. J Clin Endocrinol Metab 88:5473-5477

26. Broglio F, Gianotti L, Destefanis S, Fassino S, Abbate Daga G, Mondelli V, Lanfranco F, Gottero C, Gauna C, Hofland L, Van der Lely AJ, Ghigo E 2004 The endocrine response to acute ghrelin administration is blunted in patients with anorexia nervosa, a ghrelin hypersecretory state. Clin Endocrinol (Oxf) 60:592-599

27. Kim MS, Yoon CY, Park KH, Shin CS, Park KS, Kim SY, Cho BY, Lee HK 2003 Changes in ghrelin and ghrelin receptor expression according to feeding status. Neuroreport 14:1317-1320

28. Miljic D, Pekic S, Djurovic M, Doknic M, Milic N, Casanueva FF, Ghatei M, Popovic V 2006 Ghrelin has partial or no effect on appetite, growth hormone, prolactin, and cortisol release in patients with anorexia nervosa. J Clin Endocrinol Metab 91:1491-1495

29. Phillip M, Hershkovitz E, Rosenblum H, Savion I, Segev Y, Levy J, Frazer D 1998 Serum insulin-like growth factors I and II are not affected by undernutrition in children with nonorganic failure to thrive. Horm Res 49:76-79

30. Misra M, Miller KK, Herzog DB, Ramaswamy K, Aggarwal A, Almazan C, Neubauer G, Breu J, Klibanski A 2004 Growth hormone and ghrelin responses to an oral glucose load in adolescent girls with anorexia nervosa and controls. J Clin Endocrinol Metab 89:1605-1612

31. Barkan AL, Dimaraki EV, Jessup SK, Symons KV, Ermolenko M, Jaffe CA 2003 Ghrelin secretion in humans is sexually dimorphic, suppressed by somatostatin, and not affected by the ambient growth hormone levels. J Clin Endocrinol Metab $88: 2180-2184$ 